\title{
Predictors of Coping with Expectation Violation: An Integrative Review
}

\author{
Martin Pinquart ${ }^{1}$, Adrian Rothers ${ }^{1}$, Mario Gollwitzer ${ }^{2}$, Zahra Khosrowtaj $^{1}$, Martin $_{\text {Pietzsch }}{ }^{1}, \&$ \\ Christian Panitz ${ }^{1}$, \\ ${ }^{1}$ Philipps University, Marburg, Germany \\ ${ }^{2}$ Ludwig-Maximilians-Universität, München, Germany
}

Running head: Coping with Expectation Violation

Address all correspondence to

Martin Pinquart, Department of Psychology, Philipps University, Gutenbergstr. 18, 35032

Marburg, Germany. E-mail: pinquart@staff.uni-marburg.de

This study was conducted in the context of the Research Training Group "Expectation

Maintenance vs. Change in the Context of Expectation Violations: Connecting Different

Approaches" funded by the German Research Foundation (Deutsche Forschungsgemeinschaft, DFG Ref. no.: 290878970-GRK 2271)

Draft version $2,17 / 3 / 2021$. This paper has not yet been peer reviewed. Please do not copy or cite without author's permission. 


\begin{abstract}
The present review investigates factors that predict three processes that lead to persistence versus change of expectations after confrontation with expectation violations, based on the ViolEx (Violated Expectation) Model (Gollwitzer, Thorwart, \& Meissner, 2018) and related models. We address four groups of predictors: a) characteristics of the expectation, b) characteristics of the expectation-violating event(s), c) broader situational characteristics, and d) personality characteristics. The bulk of studies conducted in this area looked at expectation change in the direction of the experienced violation (accommodation) as their central dependent variable. The strongest empirical support was found for accommodation being less likely and minimizing of the potential impact of the discrepant information (immunization) being more likely to occur a) after the reality turns out to be worse rather than better than expected, b) if disconfirming events are more ambiguous, and c) if depressed rather than healthy people are confronted with better-thanexpected events. Given the high heterogeneity between studies on assessed predictors, we recommend a more comprehensive and unifying approach that tests the relative impact and the interplay of the whole range of predictors across paradigms.
\end{abstract}

Key words: expectation violation; expectation disconfirmation; ViolEx Model; coping 


\section{Predictors of Coping with Expectation Violation: An Integrative Review}

Expectations are beliefs about something that will occur or that will be revealed in the future (Hoorens, 2012). If expectations are disconfirmed, we speak of expectation violations. A number of models have been developed that explain why expectations are maintained or change after experiencing expectation violations: the model of coping with disconfirmed expectations by Roese and Sherman (2007), Expectancy Violations Theory (e.g., Burgoon, 2016; Afifi \& Burgoon, 2000), the Expectation-Disconfirmation Model of Consumer Satisfaction (e.g., Pieters et al., 1995), Associative Learning Theory (e.g., Rescorla \& Wagner, 1972), the Meaning Maintenance Model (Proulx \& Inzlicht, 2012), the Predictive Processing framework (e.g., Ransom et al., 2020), and the ViolEx model (Gollwitzer et al., 2018; Rief \& Glombiewski, 2016; Rief et al., 2015). Three core coping processes that contribute to persistence versus change of expectations have been identified across these models: minimization of the importance of expectation-disconfirming evidence, search for/production of future expectation-confirming evidence, and expectation change (Pinquart et al., 2021). For example, according to the ViolEx (Violated Expectations) Model, individuals may respond to expectation violations in three different ways (Gollwitzer et al., 2018; Rief \& Glombiewski, 2016). First, they may immunize their expectation against the discrepant information by ignoring it, doubting its validity, or by reformulating the expectation so that the former discrepant information can no longer disconfirm the expectation. For example, if a student received a B instead of the expected A, he or she may perceive this as an exception from the rule, or he or she may change the expectation for the next exam to simply receiving a passing grade. Second, individuals may proactively decrease the risk of future expectationdisconfirming events (assimilation). For example, after receiving a B instead on the expected A, the student may work harder for the next exam. Finally, they may change their expectation in the direction of the disconfirming event (accommodation). For example, the student may now expect to receive a $\mathrm{B}$ on the next exam, an expectation that is consistent with the 
previous grade. The ViolEx model was influenced by Brandtstädter and colleagues' model of coping with information that is inconsistent to the self-concept (e.g., Brandtstädter \& Greve, 1994; Brandtstädter, 2007).

For predicting how individuals cope with expectation disconfirmation, solid knowledge is needed regarding conditions that promote or inhibit the use ${ }^{1}$ of the strategies described above. The broad range of these predictors of assimilation, immunization, and accommodation in response to expectation violation has not yet been systematically addressed in the literature. Nonetheless, a number of assumptions can be synthetized from the literature. When focusing on responses towards expectation violations, it is self-evident that characteristics of the expectation and the expectation violation will be relevant predictors. The strength of the expectation (e.g., perceived certainty, previous confirmations, elaborateness) and the credibility of the disconfirming event (e.g., unambiguousness) are particularly important, with the former promoting persistence and the latter promoting change of the expectation (Hohwy, 2017; Ransom et al., 2020; Roese \& Sherman, 2007). Several authors have suggested that the size of the discrepancy between an a-priori expectation and an expectation-disconfirming event is a major predictor of coping with expectation violation (e.g., Afifi \& Burgoon, 2000; Roese \& Sherman, 2007). In addition, the valence of expectations and disconfirming events plays a role, as individuals tend to change their expectations more after better-than-expected events rather than worse-than-expected events (Garrett \& Sharot, 2017; Sharot et al., 2011). Human behavior, such as coping with expectation violations, is also influenced by broader characteristics of the environment (e.g., whether the expectation is shared with relevant others) and individual characteristics (Hutchinson, 2019). Relevant, individual characteristics refer to dispositions that promote

\footnotetext{
${ }^{1}$ The term "use" must not be misunderstood: we do not wish to imply that selecting a particular strategy is (typically) a rational, deliberate, strategic process. A more plausible assumption is that the cognitive processes leading individuals to prefer one strategy over another in a particular situation are often spontaneous, automatic, and uncontrollable.
} 
flexibility (accommodation) versus adherence to one's beliefs, tenacity, and avoidance of conflicting information (Brandtstädter \& Greve, 1994). Other individual characteristics, which will be discussed later, may also play a role.

Given the growing numbers of theoretical papers and empirical studies on coping with expectation disconfirmation, the goal of the present paper was to provide a comprehensive review of the literature on factors that predict the use of immunization, assimilation, and accommodation after being confronted with an expectation violation and to identify empirical support for the proposed influences on coping with expectation violations. We address inadequacies of the available research, as well as future research needs. These topics are of interest to readers from various areas of psychology. First, from a theoretical standpoint, this knowledge is relevant for the refinement and evaluation of the available models of coping with expectation violation. Second, it provides guidance for researchers regarding which potential influences on coping with expectation disconfirmation need to be analyzed more systematically due to lack of research or inconsistency between available results. Third, knowledge on predictors of coping with expectation violation is relevant for practitioners who want to change dysfunctional expectations such as those contributing to persistent mental and psychosomatic disorders (e.g., anxiety disorders, depression, chronic pain; Rief et al., 2015; Vlaeyen \& Linton, 2000), contributing to insufficient support from teachers and parents for children (Strelow et al., 2020), or contributing to negative interactions with outgroup members (Carnaghi \& Yzerbyt \&, 2007). Applying this knowledge could also increase intervention effects. For example, Doering et al. (2018) proposed expectation-focused psychotherapy to improve clinical outcomes.

In our search for relevant literature, we reviewed articles that introduced the models on coping with expectation violations and searched the PsycInfo database for studies on coping with expectation violations (search terms: expectation violation OR expectation disconfirmation OR expectancy violation OR prediction error). Based on a general model of 
predictors of coping processes by Moos and Holahan (2003), the relevant factors will be categorized into four groups: a) characteristics of the expectation, b) characteristics of the disconfirming event(s), c) broader situational characteristics, and d) personality characteristics.

\section{Characteristics of the Expectation}

Valence of the Expectation. Individuals tend to respond differently to expectation violations depending on whether they initially expected something positive or negative. For instance, in a series of learning experiments, Fazio et al. (2004) showed that expectations about negative consequences of selecting a stimulus tended to be more stable than expectations about positive consequences because expectations about negative consequences encouraged avoidance. Grupe and Nitschke (2011), as well as Dieterich et al. (2016), assessed expectations evoked by a cue that predicted an aversive or neutral picture with a $50 \%$ chance. Individuals persistently overestimated the frequency of aversive pictures, most likely due to increased attention to aversive pictures following uncertain cues.

Research on perception of others demonstrates that expectations about a person having a more favorable trait are more easily disconfirmed than expectations about having a less favorable one (Rothbart \& Bark, 1986). Changing the expectation that a target person will behave aggressively or in a hostile manner may require more and stronger disconfirming evidence than changing the expectation that a person will behave nicely and in a friendly manner. We conclude from these studies that people tend to be more willing to accommodate after violations of positive expectations compared to negative expectations due to avoidance of testing negative expectations, and/or immunizing against disconfirming information.

(Un-)Certainty of Expectations. According to the predictive processing framework, individuals with stronger expectations who are more confident, or even overconfident, are less likely to update their expectations (Benrimoh et al., 2018; Paulus et al., 2019); they will either misinterpret new information as support for their expectations or downplay disconfirming 
information (for a similar view from social psychology, see Roese \& Sherman, 2007). For example, expectations that are accompanied by a high degree of certainty tend to promote expectation-consistent perception, as shown in research on hallucinations (Benrimoh et al. 2018) and interoception (Paulus et al., 2019). Yanagisawa and Mikami (2015) experimentally manipulated the certainty of an expectation about the weight and size of an object by using foggy glasses. They showed that, in cases of higher certainty, individuals were more likely to ignore that a new stimulus differed from their expectation, thus indicating data-oriented immunization. In a learning experiment, Spicer et al. (2020) showed that following surprising events, certain expectations that were evoked by a highly predictive cue were more persistent than less certain expectations evoked by a less predictive cue.

Degree of Elaboratedness. This characteristic refers to the extent to which an initial expectation has become cognitively complex, or rich with information. Roese and Sherman (2007) suggested that in the case of weakly elaborated expectations, individuals will be more likely to ignore discrepancies between expectations and new information. More precisely, individuals may lack expectation-relevant knowledge or expertise so that expectations are vague and therefore hard to disconfirm. However, if such discrepancies are perceived, weakly elaborated expectations should be revised more readily. In contrast, the extent to which an initial expectation has become cognitively complex makes immunization strategies more likely to occur (Roese \& Sherman, 2007). To our knowledge, these assumptions have not yet been systematically tested.

Consistency of Previous Support for the Expectation. Reactions towards expectation violations are likely to depend on the ratio of remembered prior confirmations and disconfirmations. Research on the partial-reinforcement extinction effect indicates that expectations and behaviors learned under partial reinforcement are more persistent than expectations and behaviors built under continuous reinforcement (Hochman \& Erey, 2013). In other words, if individuals have experienced both confirmations and disconfirmations in the 
past, expectation violations (that is, change in the confirmation rate) will be less easily detected and less likely to lead to expectation change. In contrast, if an expectation has been constantly confirmed in the past, expectations change more rapidly after a few expectation violations.

\section{Characteristics of the Disconfirming Event(s)}

Intensity and Frequency of Expectation Disconfirmation. The model of coping with expectation disconfirmation by Roese and Sherman (2007), Expectancy Violation Theory (Afifi \& Burgoon, 2000; Burgoon, 2016), and learning theories (Rescorla \& Wagner, 1972; Niv, 2019) argue that a major predictor of coping with expectation violation is the magnitude of the discrepancy between an a-priori expectation and an expectation-disconfirming event. One-time, mild expectation-discrepant events are easier to ignore than more frequent, more discrepant events. However, there is no agreement on whether larger discrepancies lead to larger expectation change. The "Delta-rule," one of the most commonly used learning rules, assumes that larger discrepancies between expectations and disconfirming events will produce larger expectation change (Rescorla \& Wagner, 1972). Many studies on serial learning (e.g., predicting subsequent numbers in a series of presented numbers) found support for the Delta rule (Nassar et al., 2010; Yanagisawa \& Mikami, 2015).

However, Roese and Sherman (2007), proponents of the representational learning framework (Niv, 2019), as well as Kube et al. (2019), suggested that moderate discrepancies would lead to the strongest expectation change. By contrast, small discrepancies are more likely to be ignored, and large discrepancies are more likely to result in subtyping. Subtyping may be found most common if disconfirming events have some salient discriminative attributes. In fact, Seta and Seta (1994) found that mild deviation from expected behavior of a target person did not change observers' beliefs that the target person would show expectationconfirming behavior in the future, thus downplaying the expectation-violating event. In contrast, a severe deviation from expected behavior changed participants' expectation about 
how the target person specifically would behave in the future. However, they did not change expectations about how the target group would behave in general in the future. Although a specific expectation was changed, it was never generalized to expectations about other members of the target group, thus indicating subtyping. Similarly, Filipowicz et al. (2018) found that individuals were most likely to update their expectations after moderate discrepancies between expectations and events. In the case of strong discrepancies, participants seemed to base their predictions on a larger sample of previous outcomes rather than solely on the most recent (discrepant) one. When participants were asked about preconditions for updating their expectations after experiencing an expectation violation, about one quarter of them reported waiting for the same initially unexpected event to occur a number of times in quick succession. Hird et al. (2019) showed that the size of discrepancy between expected and actual pain intensity affected the intensity of pain perception. The effect of expectations on pain perceptions increased from low to medium discrepancies between expected and actual pain intensity. In the case of large discrepancies, the influence of expectation on perception decreased. Finally, Pinquart et al. (in press) found that after performing worse than expected in an achievement task, students with higher self-reported immunization scores lowered their achievement expectation for the next trial less than those with low self-reported immunization scores. However, over a series of four successive trials with achievement that was worse than expected, the expectation-stabilizing effect of immunization became weaker and then nonexistent.

Credibility of the Disconfirming Information. The Predictive Coding framework proposes that the more individuals trust the credibility of expectation-disconfirming information, the more they update their expectations accordingly (Hohwy, 2017; Ransom et al., 2020). In general, a message will be more persuasive when delivered by a trustworthy source (Hovland \& Weiss, 1951; Pilditch et al., 2020). In line with this reasoning, Kube and Glombiewski (2020b) confronted participants with projections of the global temperature rise 
from the Federal Environment Agency that were either presented as uncertain, certain, or with no additional information regarding certainty. Individuals who were skeptical about anthropogenic climate change were more likely to lower their expectations about a future temperature increase if the given information was presented as uncertain. Similarly, Kube, Rief et al. (2019) showed that feedback about success on a test on "social competence" did not affect participants' expectations about their performance in unknown tasks when they were also informed that the test's validity has not yet been established. By contrast, participants who had not received information about the validity of the test changed their expectation based on the feedback. However, experimental manipulations of the credibility of tests did not always work as expected (Kube \& Glombiewski, 2020a, b).

Unambiguousness of the Expectation-Relevant Information. Unambiguous expectation-disconfirming information may be more likely to lead to expectation change than ambiguous information because it would be more difficult to process unambiguous information in a biased way (Epley \& Kruger, 2005; Hoorens, 2002). Roese and Sherman (2007) suggested that ambiguous information is more likely to elicit the search for expectation-verifying information so that expectations persist. In fact, there is empirical evidence for interpreting ambiguous and inconsistent information (i.e., a mix of confirming and disconfirming information) as consistent with one's prior expectations (confirmation bias; e.g., Darley \& Gross, 1983; O’Brien, 2009). For example, Hoch and Ha (1986) found that consumers tend to rely on prior expectations that were based on advertising when their experience with the product was ambiguous. In contrast, consumers relied on the consumption experience after an unambiguous experience.

Valence of the Disconfirming Information/Event. If expectations predict desirable or undesirable events, disconfirming events can be better or worse than expected (Lebois et al., 2016). There is robust evidence for an asymmetrical update of expectations and beliefs after receipt of disconfirming information, colloquially referred to as the good news/bad news 
effect or optimistic reinforcement learning. Individuals are more likely to show expectation change (accommodation) in response to better-than-expected compared to worse-thanexpected events (Chowdhury et al., 2014; Garrett \& Sharot, 2017; Lefebvre et al., 2017; Sharot et al., 2011). Kube, Kirchner et al. (2019) showed that the reduced updating of expectations after worse-than-expected events can be explained by immunizing against the unexpected information. Individuals discarded worse-than-expected performance feedback by questioning its credibility and perceiving it as atypical; higher level of immunization correlated with smaller changes of the task-specific expectations.

Controllability of the Disconfirming Event(s). Pieters et al. (1995) suggested that consumers are more likely to become active in order to produce the expected outcomes if they have control over the provider's behavior. Otherwise, individuals will change their expectations in response to the expectation violation. Similarly, Dalal and Agrawal (1987) showed that after failing on an examination, individuals reduced the expectations about their achievement in a future examination of a similar nature more if they perceived the source of failure as stable and uncontrollable.

\section{Broader Situational Characteristics}

Similarity of Expectation to Those of Relevant Others. Carolan (2017) suggested that violated expectations will change less if significant others share the same expectations. For example, if a group of high school friends expects to go to college, unexpected bad grades will not likely lead to abandoning the expectation of enrolling in college. In fact, a study showed that an increase in exposure to peer drinkers (who have positive expectations about alcohol consumption) led to an increase in positive expectations about alcohol use (Sadler, 2005). An experiment by Carnaghi and Yzerbyt (2007) showed that observers preserved consensual beliefs about a group after being confronted with counter-stereotypic group members. They were more likely to perceive people who behaved in an unexpected, counterstereotypic way as atypical members of the group when the stereotype was shared among an 
ingroup. In a study on processing information about a target person's guilt, individuals were more likely to interpret ambiguous information in line with their expectation if they believed that their expectation was shared by another person (Willard, 2008).

Cognitive Load. Cognitive load refers to the working memory resources in use. Individuals will have less working memory resources available for coping with expectation disconfirmation if they simultaneously perform a second task. There is some controversy as to whether a higher cognitive load leads to less or more attention for expectation-disconfirming information while the ability for deeper cognitive processing is inhibited (see, Sherman et al., 1998). A number of experiments by Sherman et al. (1998) showed that expectationdisconfirming (stereotype-disconfirming) information evoked stronger attentional processing than consistent information when cognitive resources were depleted. This indicates that ignoring expectation-disconfirming information will be inhibited. However, a high cognitive load also inhibited deeper encoding of disconfirming information. Thus, cognitively demanding forms of immunization, such as searching for reasons why the disconfirming event was an exception from the rule, will also be inhibited.

Prior Coping with the Disconfirming Information. Only one mode of coping with an expectation violation may not be sufficient if, for example, discrepant information cannot persistently be ignored. Therefore, researchers proposed a typical sequence of coping behaviors. Greve (2007) suggested that individuals react with immunizing processes first (perceptual avoidance, avoidance of acceptance of the information, immunization). If immunization fails, individuals assimilate or accommodate their expectations. With regard to the latter processes, Brandtstädter and Greve (1994) suggest that the motivation to accommodate increases when confidence in assimilation is eroded by repeated failure.

Mili (2020) found that after expectation violation, students tended to react with assimilation first, followed by accommodation of their expectations if assimilation did not lead to future expectation confirmation. As too few students had reported immunization in 
that study, the author could not test Greve's claim that immunization may typically precede assimilation (Greve, 2007). Accommodation and assimilation may also be shown in parallel. For example, after disconfirmation of high achievement expectations in a prior exam, students reduced their achievement expectations and increased their effort and related performance in the next exam (Radhakrishnan et al., 1996).

\section{Personality Characteristics}

Goal-related Coping Dispositions. The ViolEx model's terms of accommodation, assimilation, and immunization are borrowed from Brandtstädter's model on coping with information that is discrepant to the self-concept (e.g., Brandtststädter, 2007; Brandtstädter \& Greve, 1994). Brandtstädter assessed individual dispositions in coping with discrepancies between actual and desired states, such as unattained goals (Brandtstädter \& Renner, 1990). Individuals with high levels of tenacious goal pursuit (i.e., tenacity) show goal-focused resource mobilization and compensatory efforts to attain the goal. Flexible goal adjustment (i.e., flexibility), on the other hand, describes the tendency to employ accommodative strategies in the face of goal-outcome discrepancies. This means that individuals high in flexibility more readily adapt their goals and goal-related expectations. They also show "palliative" responses to reduce the aversiveness of failed goal attainment, for example by concentrating on positive aspects of the negative event. While Brandtstädter's questionnaire addresses coping with goal attainment failures rather than expectation violations, the coping dimensions of his model and the ViolEx model are similar to each other. Pinquart et al. (in press) found a small, albeit significant association of children's self-reported assimilative coping with violated achievement expectations and parental reports on their child's dispositional tenacious goal pursuit. Whether significant results would also be found in adult samples remains to be tested.

Cognitive Preferences and Styles. Humans rely on their ability to structure information about the world into expectations, schemas, and rules that are simplified models 
of reality. This ability varies reliably between individuals, which is reflected by cognitive styles or epistemic preferences such as personal need for structure (the preference for simple cognitive structures; Neuberg \& Newsom, 1993), need for cognitive closure (the desire for clear answers as opposed to ambiguity; Kruglanski \& Webster, 1996), and (in-)tolerance for ambiguity (viewing ambiguous stimuli in a neutral and open way or as a threat; Budner, 1962). Assessments of these traits are moderately to highly correlated (Neuberg et al., 1997). In theory, higher trait levels might predispose individuals to ignore and resist expectationinconsistent information (i.e., immunization, assimilation) in order to protect their models of the world (Neuberg \& Newsom, 1993). In fact, individuals with a higher need for closure paid less attention to and were less likely to remember behavior that was inconsistent with their initial expectation (Dijksterhuis et al., 1996). When having the opportunity to choose between information that supports or challenges one's decision to extend or end the contract of a manager, individuals with a higher need for cognitive closure showed a stronger tendency to pay attention to information that supported rather than challenged their expected decision (Hart et al., 2012).

However, more recent research demonstrates that the effects of a need for structure or cognitive closure are context-dependent. Selective attention for expectation-consistent information is mainly promoted when individuals have previously built explicit categories and were encouraged to engage in categorical processing (Dijksterhuis et al., 1996), and when available cognitive resources for information processing are limited (Strojny et al., 2016). Otherwise, a high need for structure or cognitive closure may even lead to an increase in both searching for and processing expectation-inconsistent information, possibly because thinking about such information might allow it to be reconciled with the expectation (Kemmelmeier, 2015; Strojny et al., 2016).

Preference for consistency is only weakly associated with the constructs discussed in the previous paragraph. It describes the individual's desire for their beliefs, attitudes, 
perceptions, and behaviors to be congruent and consistent (Cialdini et al., 1995). With regard to expectations, Alós-Ferrer et al. (2016) used a risky-choice paradigm with monetary rewards to investigate the association between preference for consistency and decision inertia (i.e., sticking to a decision independent of decision outcomes). In each trial, participants took a ball from one of two urns that allowed for a possibility to win money, as well as a probabilistic inference on whether participants should stick with the chosen urn or change the urn for a second drawing. The authors found that individuals with a higher preference for consistency chose to stay with their first choice more often, even when changing urns would increase their probability of winning. In other words, participants with a high preference for consistency were less prone to show accommodation of their reward expectations associated with their choice. Thus, preference for consistency seems to inhibit accommodation and/or promote immunization of expectations in order to avoid doubts about the appropriateness of previous behavior.

Positive and Negative Affectivity. As the valence of expectations and disconfirming outcomes can be critical in influencing the selection of coping mechanisms, stable individual differences in the susceptibility to valent information may moderate the selection of coping strategies. In the case of positive affectivity, biased coping with expectation violations may contribute to stronger accommodation, better-than-expected outcomes, and/or stronger immunization against worse-than-expected outcomes. A very prominent positive affectivity trait relevant to expectations is optimism, the stable disposition to have positive outcome expectations that generalize across situations (Scheier \& Carver, 1985). A recent study found that, after getting worse-than-expected achievement feedback, participants with high optimism scores showed a trend of updating their performance expectations less than those who scored low on optimism (Kube \& Glombiewski, 2020b). In contrast, optimism did not predict stronger accommodation to better-than-expected feedback nor weaker accommodation to worse-than-expected feedback in a study by Korn et al. (2014). 
Optimism has repeatedly been associated with stronger effects of analgesic placebo expectations (Darragh et al., 2015; Kern et al., 2020). Importantly, stronger placebo effects among optimists might not only be caused by more positive expectations, but also by more efficient immunization against negative expectation violations. For example, it was found that - in contrast to optimist in the control condition or pessimists - only optimistic individuals who received analgesic expectation instructions reported less pain in the cold pressor task. In addition, dispositional optimism did not affect initial expectations about the pain intensity before immersing the hand into an ice water container. Thus, initial expectations did not mediate the effect of optimism on later pain judgments, supporting the suggested role of optimism for immunization against pain stimuli (Geers et al., 2010). Affective expectation research also provides evidence for more immunization tendencies in optimists, both for outcomes that are worse and better than expected. In one study, optimists who were promised a funny video, but were shown a boring one rated the video as more positive than optimists who received no a priori information or pessimists. They also rated a video less positively when it was announced as boring but was rather funny (Geers \& Lassiter, 2002). In a different study, participants had to judge whether the intensity of an emotional expression on the screen matched a numeric intensity rating that was given beforehand by the computer. Optimists tended to agree more often with the prediction, regardless of the depicted emotion type (happiness, sadness, fear) and the magnitude of discrepancy (Morton et al., 2011).

On the other side of the affective spectrum, increased neuroticism, trait anxiety, and major depression have been associated with biased reactions towards expectation violations (Aue \& Okon-Singer, 2015). One could suspect that highly neurotic, anxious, and depressed individuals (a) accommodate their expectations more strongly to worse-than-expected outcomes and/or (b) immunize more strongly when negative expectations are disconfirmed by better-than-expected outcomes. In line with the latter assumption, Pavlovian fear conditioning studies have shown that individuals high in neuroticism or trait anxiety show extinction 
resistance once the previously threat-inducing cue is not followed by the aversive stimulus anymore. This indicates less accommodation of expectations about consequences of the former threat-inducing cue (e.g., Gazendam et al., 2015; Staples-Bradley et al., 2018). However, traits that reflect a dispositional cognitive dissonance between a positive hope and a negative expectation (e.g., rejection sensitivity, victim sensitivity) yield a different pattern: Participants high in victim sensitivity (i.e., a trait reflecting a strong need to trust others, yet a dispositional expectation that other people are untrustworthy) accommodate their social expectations more strongly to better-than-expected outcomes than people low in victim sensitivity, for example, when a target person behaves in a more friendly manner than expected (Süssenbach et al., 2016).

Turning to dispositional anxiety, individuals suffering from anxiety disorders take longer to reduce fear responding to former threat cues even when these cues do not predict harm anymore (e.g., Duits et al., 2015). Meanwhile, fear conditioning research does not support stronger accommodation to worse-than-expected outcomes in individuals with high trait anxiety or neuroticism per se; fear conditioning paradigms typically employ unambiguous and highly predictable threats to which individuals adapt their expectations regardless of their trait anxiety/neuroticism levels (Lonsdorf \& Merz, 2017). In research on academic achievement, a series of studies investigated the role of trait anxiety on a surprising violation of one's performance expectations. They found that anxious individuals both decreased performance expectations after negative feedback more than low anxious individuals and increased them more than low anxious individuals after surprising positive feedback (Rychlak \& Lerner, 1965; Shimkunas, 1970).

The diverging results of decreased accommodation in fear extinction research versus increased accommodation of performance expectations after better-than-expected outcomes are most likely explained by differences in expectation, confidence, and stability; fear extinction resistance has been observed after individuals have already completed several trials 
of fear conditioning. In contrast, the observed increased accommodation was based on studies where individuals worked on a performance task for the first time and in very few trials.

A series of experiments showed that in contrast to healthy people, depressed individuals did not accommodate their negative performance expectations to positive performance feedback (Korn et al., 2014; Kube \& Glombiewski, 2020a; Kube, Rief et al., 2019). The authors attributed this to data-oriented immunization because providing cues that promoted immunization inhibited an update after the receipt of better-than-expected feedback while providing immunization-inhibiting cues promoted expectation change (Kube, Glombiewski et al., 2019; Kube, Rief et al., 2019).

Metatraits of Maintenance versus Change. A global perspective on personality and responding to expectation violation is provided by traits with broad transsituational application, such as the Big Five metatraits Stability and Plasticity (DeYoung, 2015). According to DeYoung's Cybernetic Big Five theory, individuals with high Stability levels (i.e., Emotional Stability, Conscientiousness, and Agreeableness) tend to show more behavior aimed at protecting goals, interpretations, and strategies. In contrast, high levels of Plasticity (i.e., Extraversion and Openness) predict more explorative behavior, such as creating new goals, interpretations, and strategies. Studies on associations between these metatraits and other variables do not (yet) include investigations on mechanisms of expectation maintenance versus change. One may speculate that high levels of Stability are related to low levels of accommodation, as well as high levels of avoidance of expectation-disconfirming events and immunization against received discrepant information. It is less clear whether Plasticity predicts engagement in active behavior to approach expectation-confirming events, which would be a form of assimilative behavior, or higher accommodation of one's expectations by approaching expectation-disconfirming events.

\section{Discussion}

The present review shows that many factors contribute to the ways of coping with 
expectation violations (see Table 1 for summary). Most evidence is available for reduced accommodation and increased immunization of expectations a) after the reality turns out to be worse than expected rather than better, b) if disconfirming events are more ambiguous, and c) if depressed rather than healthy people are confronted with better-than-expected events. However, even here the empirical evidence is based on a small number of studies. It should be mentioned that higher availability of empirical research for the three listed variables does not necessarily mean that these variables are also the strongest statistical predictors of how individuals cope with expectation violations, as some potential predictor variables have not yet been systematically assessed. Until now, many predictors have been analyzed mainly in one specific context, such as performance on achievement tests (e.g., goal-related coping dispositions) or fear conditioning (e.g., neuroticism and anxiety). In addition, we noted a complete lack of empirical studies on some theoretically plausible predictors, such as dispositional use of the strategies of coping with expectation violation, and personality metatraits.

In order to gain reliable data on the relative effects of predictor variables, standardized expectation violation paradigms are required, in which an extensive list of predictor variables is tested. In a first step, expectations could be induced and violated via adaptations of wellestablished paradigms (e.g., Kube \& Glombiewski, 2020a, b; Süssenbach et al., 2016). Researchers should include an extensive list of predictor variables rather than a single predictor because some of the predictors tend to be correlated. While this has already been shown with regard to different cognitive preferences and styles (e.g., Neuberg et al., 1997), perceived certainty of an expectation, degree of elaboratedness, and the amount of previous expectation confirmation are most likely correlated as well (Jepma et al., 2020).

Simultaneously including these variables will provide insights into which of them best predicts persistence versus change of expectations, as well as the coping processes that contribute to persistence and change. In addition, they should directly assess all of the coping 
variables proposed by the ViolEx model and related models in order to separate effects of predictors on the different ways of coping. After testing the wide range of potential influences on coping within a unified research paradigm, subsequent studies should test whether the results on expectations in one domain can be generalized to different domains of expectations.

It is important for studies to also assess the interplay of situational and trait predictor variables, as positivity of the discrepant information and dispositional optimism (Korn et al., 2014), as well as depressive symptoms (Korn et al., 2014; Kube, Rief et al., 2019), have been shown to interact. The contradictory theoretical assumptions and empirical results on whether large discrepancies between expectations and disconfirming events lead to large expectation change (Nassar et al., 2010; Rescorla \& Wagner, 1972; Yanagisawa \& Mikami, 2015) or immunization against the discrepant information and persistence of the expectation (Filipowicz et al., 2018; Kube et al., 2019; Niv, 2019; Roese and Sherman, 2007; Seta \& Seta, 1994) call for more attention to the boundary conditions for effects of predictor variables. For example, personality characteristics may have larger effects on the ways of coping with expectation violations if the discrepant information is more ambiguous.

While studies on event characteristics most often employed an experimental design that provides information on causality, there were mostly non-experimental, correlational studies on the association of personality characteristics with coping with expectation violations. Although some variables such as personality traits cannot be experimentally manipulated, well-designed quasi-experiments that eliminate as many threats to internal validity as possible are needed (Leary, 2001). Thus, we recommend that strong, internally valid empirical designs are used in future research on factors that causally predict the occurrence of coping strategies.

Most available studies on predictors of coping with expectation violations assessed accommodation, while very few studies measured assimilation (Table 1). Many researchers may have been more interested in expectation change than in the persistence of expectations 
and related processes of assimilation and immunization that contribute to persistence. In addition, as accommodation indicates expectation update by definition, it can be easily assessed by retesting expectations compared to other strategies for which separate measures have to be developed. The seeming lack of studies on assimilation can be explained by the fact that assimilative behavior after the experience of an expectation violation is focused on the next cycle of expectation (dis)confirmation and is, therefore, only relevant when similar events repeatedly occur over time. In addition, this behavior will only be relevant if individuals expect to have some control over the occurrence of the future event (Pieters et al., 1995; Dalal \& Agrawal. 1987). Finally, specific assimilative behaviors have to be measured according to the violated individual expectation, such as making more efforts for preparing the next exam or trying to change the behavior of a friend. This makes the assessment of assimilation more challenging than the assessment of accommodation, and some forms of immunization as well such as interpreting the expectation violation as an exception to the rule. In regards to getting more knowledge about effects of predictor variables on the whole range of coping strategies, more efforts are needed for developing assessments of assimilation and immunization, and applying these measures simultaneously in studies of coping with expectation violations.

The present review focused on four groups of influences on coping with expectation violations. We did not address whether coping may also vary by sociodemographic characteristics. Some research is available on age differences, but the results are difficult to generalize as different expectations have been assessed in different age groups, measures of coping differed across studies (e.g., Chowdhury et al., 2017; Pinquart \& Block, 2020), and most studies addressed only a narrow age range. In principle, the same assessments could be applied from mid-adolescence until old age. In contrast, adaptations or the development of unique assessment methods are necessary when working with children with lower verbal and cognitive skills (e.g., Lessing et al., 2019). The present review also did not address genetic 
influences, which build a biological base for personality differences in coping with expectations violation (see, for example, the study on the association of the dopaminergic COMT val158met polymorphism with susceptibility to expectation confirmation bias by Doll et al., 2011).

In sum, scientific knowledge on factors that affect how people cope with expectation violations has been growing recently, with almost two thirds of the cited studies conducted in the last decade. We conclude that the most consistent findings are available with regard to a) a selective update of expectations after better-than-expected rather than worse-than expected events, b) a stronger update in response to unambiguous expectation disconfirmations, and c) an inhibition of expectation change if depressed individuals experience better-than-expected events. Nonetheless, we need more unifying research paradigms to test the whole range of potential predictors. As shown in the present review, the available research provides valuable insights in which factors affect coping with expectation violations and should therefore be included in future large-scale studies.

Future research should a) provide a systematic test of the broad range of potential influences on coping with expectation violations and their interplay, b) use this empirical knowledge for the refinement of the theoretical models in that field, and c) apply this knowledge for the development of interventions aimed at changing persistent dysfunctional expectations, such as those contributing to persistent mental and psychosomatic disorders (Doering et al., 2018; Rief et al., 2015; Vlaeyen \& Linton, 2000) or negative stereotypes about outgroups (Carnaghi \& Yzerbyt \&, 2007). 


\section{References}

Afifi, W. A., \& Burgoon, J. K. (2000). The impact of violations on uncertainty and consequences for attractiveness. Human Communication Research, 26, 203-233. doi:10.1111/j.1468-2958.2000.tb00756.x

Alós-Ferrer, C., Hügelschäfer, S., \& Li, J. (2016). Inertia and decision making. Frontiers in Psychology, 7, 169. doi:10.3389/fpsyg.2016.00169

Aue, T., \& Okon-Singer, H. (2015). Expectancy biases in fear and anxiety and their link to biases in attention. Clinical Psychology Review, 42, 83-95. doi:10.1016/j.cpr.2015.08.005

Benrimoh, D., Parr, T., Vincent, P., Adams, R. A., \& Friston, K. J. (2018). Active inference and auditory hallucinations. Computational Psychiatry, 2, 183-204. doi:10.1162/cpsy_a_0002

Brandtstädter, J. (2007). Das flexible Selbst: Selbstentwicklung zwischen Zielbindung und Ablösung [The flexible self: Self-development between target commitment and detachment]. München: Elsevier.

Brandtstädter, J., \& Greve, W. (1994). The aging self: Stabilizing and protective processes. Developmental Review, 14, 52-80. doi:10.1006/drev.1994.1003

Brandtstädter, J., \& Renner, G. (1990). Tenacious goal pursuit and flexible goal adjustment: Explication and age-related analysis of assimilative and accommodative strategies of coping. Psychology and Aging, 5, 58-67. doi:10.1093/ageing/afv203

Budner, S. (1962). Intolerance of ambiguity as a personality variable. Journal of Personality, 30, 29-50. doi:10.1111/j.1467-6494.1962.tb02303.x

Burgoon, J. K. (2016). Expectancy violations theory. In C. R. Berger, M.E. Roloff, S. R. Wilson, J. P. Dillard, J. Caughlin, \& D. Solomon (eds.), International encyclopedia of interpersonal communication (pp. 1-9). Hoboken, NJ: Wiley.

Carnaghi, A., \& Yzerbyt, V. Y. (2007). Subtyping and social consensus: The role of the 
audience in the maintenance of stereotypic beliefs. European Journal of Social Psychology, 37, 902-922. doi:10.1002/ejsp.402

Carolan, B. V. (2017). Assessing the adaptation of adolescents' educational expectations: Variations by gender. Social Psychology of Education, 20, 237-257. doi:10.1007/s11218-017-9377-y

Chowdhury, R., Sharot, T., Wolfe, T., Düzel, E., \& Dolan, T.J. (2017). Optimistic update bias increases in older age. Psychological Medicine, 44, 2003-2012. doi:10.1017/S0033291713002602

Cialdini, R. B., Trost, M. R., \& Newsom, J. T. (1995). Preference for consistency: The development of a valid measure and the discovery of surprising behavioral implications. Journal of Personality and Social Psychology, 69, 318-328. doi:10.1037/0022-3514.69.2.318

Cumsille, P. E., Sayer, A. G., \& Graham, J. W. (2000). Perceived exposure to peer and adult drinking as predictors of growth in positive alcohol expectancies during adolescence. Journal of Consulting and Clinical Psychology, 68, 531-536. doi:10.1037/0022006X.68.3.531

Dalal, A. K., \& Agrawal, R. (1987). Causal thinking and the expectation for success in the achievement context. Journal of General Psychology, 114, 57-68. doi:10.1080/00221309.1987.9711055

Darley, J. M., \& Gross, P. H. (1983). A hypothesis-confirming bias in labeling effects. Journal of Personality and Social Psychology, 44, 20-33. doi:10.1037/00223514.44 .1 .20

Darragh, M., Booth, R. J., \& Consedine, N. S. (2015). Who responds to placebos? Considering the "placebo personality" via a transactional model. Psychology, Health and Medicine, 20, 287-295. doi: 10.1080/13548506.2014.936885

DeYoung, C. G. (2015). Cybernetic Big Five Theory. Journal of Research in Personality, 56, 
COPING WITH EXPECTATION VIOLATION 25

33-58. doi:10.1016/j.jrp.2014.07.004

Dieterich, R., Endrass, T., \& Kathmann, N. (2016). Uncertainty is associated with increased selective attention and sustained stimulus processing. Cognitive \& Affective Behavioral Neuroscience, 16, 447-456. doi:10.3758/s13415-016-0405-8

Dijksterhuis, A., van Knippenberg, A., Kruglanski, A. W., \& Schaper, C. (1996). Motivated social cognition: Need for closure effects on memory and judgment. Journal of Experimental Social Psychology, 32, 254-270. doi:10.1006/jesp.1996.0012

Doering, B., Glombiewski, J. A., \& Rief, W. (2018). Expectation-focused psychotherapy to improve clinical outcomes. International Review of Neurobiology, 138, 257-270. doi:10.1016/bs.irn.2018.02.004

Doll, B. B., Hutchison, K. E., \& Frank, M. J. (2011). Dopaminergic genes predict individual differences in susceptibility to confirmation bias. Journal of Neuroscience, 31, 61886198. doi:10.1523/JNEUROSCI.6486-10.2011

Duits, P., Cath, D. C., Lissek, S., Hox, J. J., Hamm, A. O., Engelhard, I. M., ... Baas, J. M. P. (2015). Updated meta-analysis of classical fear conditioning in the anxiety disorders. Depression and Anxiety, 32, 239-253. doi:10.1002/da.22353

Epley, N., \& Kruger, J. (2005). When what you type isn't what they read: The perseverance of stereotypes and expectancies over e-mail. Journal of Experimental Social Psychology, 41, 414-422. doi:10.1016/j.jesp.2004.08.005

Fazio, R. H., Eiser, J. R., \& Shook, N. J. (2004). Attitude formation through exploration: Valence asymmetries. Journal of Personality and Social Psychology, 87, 293-311. doi:10.1037/0022-3514.87.3.293

Filipowicz, A., Valadao, D., Anderson, B., \& Danckert, J. (2018). Rejecting outliers: Surprising changes do not always improve belief updating. Decision, 5, 165-176. doi:10.1037/dec0000073

Garrett, N., \& Sharot, T. (2017). Optimistic update bias holds firm: Three tests of robustness 
following Shah et al. Consciousness and Cognition, 50, 17-22. doi:10.1016/j.concog.2016.10.013

Gazendam, F. J., Kamphuis, J. H., Eigenhuis, A., Huizenga, H. M. H., Soeter, M., Bos, M. G. N., ... Kindt, M. (2015). Personality predicts individual variation in fear learning. Clinical Psychological Science, 3, 175-188. doi:10.1177/2167702614535914

Geers, A. L., Wellman, J. A., Fowler, S. L., Helfer, S., \& Frances, C. R. (2010). Dispositional optimism predicts placebo analgesia. Journal of Pain, 11(11), 1165-1171. doi:10.1016/j.jpain.2010.02.014

Geers, A. L., \& Lassiter, G. D. (2002). Effects of affective expectations on affective experience: The moderating role of optimism-pessimism. Personality and Social Psychology Bulletin, 28, 1026-1039. doi:10.1177/01461672022811002

Gollwitzer, M., Thorwart, A., \& Meissner, K. (2018). Psychological responses to violations of expectations. Frontiers in Psychology, 8, 2357. doi:10.3389/fpsyg.2017.02357

Greve, W. (2007). Das erwachsene Selbst [The adult self]. In W. Greve (Ed.), Psychologie des Selbst (pp. 96-131). Weinheim: Beltz.

Grupe, D. W., \& Nitschke, J. B. (2011). Uncertainty is associated with biased expectancies and heightened responses to aversion. Emotion, 11, 413-424. doi:10.1037/a0022583

Hallett, J., McManus, A., Maycock, B. R., Smith, J., \& Howat, P.M. (2014). “Excessive drinking — an inescapable part of university life?” A focus group study of Australian undergraduates. Open Journal of Preventive Medicine, 4, 616-629. doi:10.4236/ojpm.2014.47071

Hart, W., Adams, J. M., Burton, K. A., Shreves, W., \& Hamilton, J. C. (2012). Shaping reality vs. hiding from reality: Reconsidering the effects of trait need for closure on information search. Journal of Research in Personality, 46, 489-496. doi:10.1016/jrp.2012.05.004

Hird, E. J., Charalambous, C., El-Deredy, W., Jones, A. K., \& Talmi, D. (2019). Boundary 
effects of expectation in human pain perception. Scientific Reports, 9, 9443. doi:10.1038/s41598-019-45811-x

Hoch, H. J., \& Ha, Y. W. (1986). Consumer learning: Advertising and the ambiguity of product experience. Journal of Consumer Research, 13, 221-233. doi:10.1086/209062

Hochman, G., \& Erey, I. (2013). The partial-reinforcement extinction effect and the contingent-sampling hypothesis. Psychonomic Bulletin and Review, 20, 1336-1342. doi:10.3758/s13423-013-0432-1

Hohwy, J. (2017). Priors in perception: Top-down modulation, Bayesian perceptual learning rate, and prediction error minimization. Consciousness and Cognition, 47, 75-85. doi:10.1016/j.concog.2016.09.004

Hoorens, V. (2012). Expectations. In V. Ramachandran (Ed.), Encyclopedia of Human Behavior (2 ${ }^{\text {nd }}$ ed., pp. 142-149). Amsterdam: Elsevier.

Hovland, C. I., \& Weiss, W. (1951). The influence of source credibility on communication effectiveness. Public Opinion Quarterly, 15, 635-650. doi:10.1086/266350

Hutchinson, E. D. (2019). Dimensions of human behavior: Person and environment ( $6^{\text {th }}$ ed.). Thousand Oaks, CA: Sage.

Jepma, M., Schaaf, J. V., Visser, I., \& Huizenga, H. M. (2020). Uncertainty-driven regulation of learning and exploration in adolescents: A computational account. PLoS Computational Biology, 16(9), e1008276. doi:10.1371/journal. pcbi.1008276

Kemmelmeier, M. (2015). The closed-mindedness that wasn't: Beed for structure and expectancy-inconsistent information. Frontiers in Psychology, 6, 896. doi:10.3389/fpsyg.2015.00896

Kern, A., Kramm, C., Witt, C. M., \& Barth, J. (2020). The influence of personality traits on the placebo/nocebo response: A systematic review. Journal of Psychosomatic Research, 128, 109866. doi:10.1016/j.jpsychores.2019.109866

Korn, C. W., Sharot, T., Walter, H., Heekeren, H. R., \& Dolan, R. J. (2014). Depression is 
related to an absence of optimistically biased belief updating about future life events. Psychological Medicine, 44, 579-592. doi:10.1017/S0033291713001074

Kube, T., \& Glombiewski, J. A. (2020a). How depressive symptoms hinder positive information processing - An experimental study on the interplay of cognitive immunisation and negative mood in the context of expectation update. Cognitive Therapy and Research. doi:10.31234/osf.io/cegwh

Kube, T., \& Glombiewski, J. A. (2020b, July 23). How reappraisal of new information contributes to optimistic belief updating. doi:10.31219/osf.io/be5vx

Kube, T., Glombiewski, J. A., Gall, J., Touissant, L., Gärtner, T. G., \& Rief, W. (2019). How to modify persisting negative expectations in major depression? An experimental study comparing three strategies to inhibit cognitive immunization against novel positive experiences. Journal of Affective Disorders, 250, 231-240. doi:10.1016/j.jad.2019.03.027

Kube, T., Kirchner, L., Rief, W., Gärtner, T., \& Glombiewski, J. A. (2019). Belief updating in depression is not related to increased sensitivity to unexpectedly negative information. Behaviour Research and Therapy, 123, 103509. doi:10.1016/j.brat.2019.103509

Kube, T., Rief, W., Gollwitzer, M., Gärtner, T., \& Glombiewski, J. A. (2019). Why dysfunctional expectations in depression persist - Results from two experimental studies investigating cognitive immunization. Psychological Medicine, 49, 15321544. doi:10.1017/S0033291718002106

Kube, T., Schwarting, R., Rozenkrantz, L., Glombiewski, J. A., \& Rief, W. (2020). Distorted cognitive processes in major depression: A predictive processing perspective. Biological Psychiatry, 87, 388-398. doi:10.1016/j.biopsych.2019.07.017

Kruglanski, A. W., \& Webster, D. M. (1996). Motivated closing of the mind: "Seizing" and “freezing." Psychological Review, 103, 263-283. doi:10.1037/0033-295X.103.2.263 Leary, M. R. (2001). Introduction to behavioral research methods. Boston: Allyn \& Bacon. 
Lebois, L. A. M., Hertzog, C., Slavich, G. M., Barrett, L. F., \& Barsalou, L. W. (2016). Establishing the situated features associated with perceived stress. Acta Psychologia, 169, 119-132. doi:10.1016/j.actpsy.2016.05.012

Lefebvre, G., Lebreton, M., Meyniel, F., Bourgeois-Gironde, S., \& Palminteri, S. (2017). Behavioural and neural characterization of optimistic reinforcement learning. Nature Human Behavior, 1, 0067. doi:10.1038/s41562-017-0067

Lessing, N., Kappes, C., Greve, W., \& Mähler, C. (2019). Developmental conditions of accommodative coping in childhood: The role of executive functions. Cognitive Development, 50, 56-65. doi:10.1016/j.cogdev.2019.02.002

Lonsdorf, T. B., \& Merz, C. J. (2017). More than just noise: Inter-individual differences in fear acquisition, extinction and return of fear in humans - Biological, experiential, temperamental factors, and methodological pitfalls. Neuroscience and Biobehavioral Reviews, 80, 703-728. doi:10.1016/j.neubiorev.2017.07.007

Mili, A. (2020). Umgang mit Erwartungsverletzungen bei Studierenden [Coping with expectation violations in students]. Unpublished bachelor thesis, University of Marburg.

Moos, T. H., \& Holahan, C.J. (2003). Dispositional and contextual perspectives on coping: Toward an integrative framework. Journal of Clinical Psychology, 59, 1387-1403. doi:10.1002/jclp.10229

Morton, D. L., El-Deredy, W., Morton, A. S., Elliott, R., \& Jones, A.K. P. (2011). Optimism facilitates the utilisation of prior cues. European Journal of Personality, 25, 424-430. doi:10.1002/per.805

Nassar, M.R., Wilson, R.C., Heasly, B., \& Gold, J. (2010). An approximately Bayesian DeltaRule model explains the dynamics of belief updating in a changing environment. Journal of Neuroscience, 30, 12366-12378. doi:10.1523/JNEUROSCI.0822-10.2010

Neuberg, S. L., Judice, T. N., \& West, S. G. (1997). What the need for closure scale measures and what it does not: Toward differentiating among related epistemic motives. 
Personality Processes and Individual Differences, 72, 1396-1412. doi:10.1037/00223514.72.6.1396

Neuberg, S. L., \& Newsom, J. T. (1993). Personal need for structure: Individual differences in the desire for simple structure. Journal of Personality and Social Psychology, 65, 113131. doi: $10.1037 / 0022-3514.65 .1 .113$

Niv, Y. (2019). Learning task-state representations. Nature Neuroscience, 22(10), 1544-1553. doi:10.1038/s41593-019-04708

O’Brien, B. (2009). Prime suspect: An examination of factors that aggravate and counteract confirmation bias in criminal investigations. Psychology, Public Policy, and Law, 15, 315-334. doi:10.1037/a00178

Paulus, M. P., Feinstein, J. S., \& Khalsa, S. S. (2019). An active inference approach to interoceptive psychopathology. Annual Review of Clinical Psychology, 15, 97122.doi:10.1146/annurev-clinpsy-050718-095617

Pilditch, T. B., Madsen, J. K., \& Custers, R. (2020). False prophets and Cassandra's curse: The role of credibility in belief updating. Acta Psychologica, 202, 102956. doi:10.1016/j.actpsy.2019.102956

Pieters, R., Koelmeijer, K., \& Roest, H. (1995). Assimilation processes in service satisfaction formation. International Journal of Service Industry Management, 6, 17-33.

Pinquart, M. \& Block, H. (2020). Coping with broken achievement-related expectations in students from elementary school: An experimental study. International Journal of Developmental Science, 14, 9-17. doi:10.3233/DEV-200001

Pinquart, M., Endres, D., Teige-Mocigemba, S., Panitz, C., \& Schütz, A. C. (2021). Why expectations do or do not change after expectation violation: A comparison of six models. Consciousness and Cognition, 89, 103086. doi:10.1016/j.concog.2021.103086 Pinquart, M., Koß, J.C., \& Block, H. (in press). How students react when their performance is worse or better than expected? An analysis based on the ViolEx Model. Zeitschrift für 
Entwicklungspsychologie und Pädagogische Psychologie. doi:10.1026/0049$8637 / \mathrm{a} 00022$

Proulx, T., \& Inzlicht, M. (2012). The five "A“"s of meaning maintenance: Finding meaning in the theories of sense-making. Psychological Inquiry, 23, 317-335. doi:10.1080/1047840x.2012.702372

Rescorla, R. A., \& Wagner, A. R. (1972). A theory of Pavlovian conditioning: Variations in the effectiveness of reinforcement and non-reinforcement. In A. H. Black \& W. F. Prokasy (Eds.), Classical Conditioning II: Current Theory and Research (pp. 64-99). New York: Appleton-Century Crofts.

Rief, W., \& Glombiewski, J.A. (2016). Expectation-focused psychological interventions. Verhaltenstherapie, 26, 47-54. doi:10.1159/000442374

Rief, W., Glombiewski, J. A., Gollwitzer, M., Schubö, A., Schwarting, R., \& Thorwart, A. (2015). Expectations as core features of mental disorders. Current Opinions in Psychiatry, 28, 378-385. doi:10.1097/YCO.0000000000000184

Roese, N. J., \& Sherman, J. W. (2007). Expectancy. In E. T. Higgins \& A. W. Kruglanski (Eds.), Social Psychology: Handbook of Basic Principles (2 ${ }^{\text {nd }}$ ed., pp. 91-115). New York: Guilford.

Rothbart, M., \& Park, B. (1986). On the confirmability and disconfirmability of trait concepts. Journal of Personality and Social Psychology, 50, 131-142. doi:10.1037/00223514.50 .1 .131

Rychlak, J. F., \& Lerner, J. J. (1965). An expectancy interpretation of manifest anxiety. Journal of Personality and Social Psychology, 2, 677-684. doi:10.1037/h0022627

Scheier, M. F., \& Carver, C. S. (1985). Optimism, coping, and health: Assessment and implications of generalized outcome expectancies. Health Psychology, 4, 219-247. doi:10.1037/0278-6133.4.3.219

Seta, J. S., \& Seta, C. E. (1994). Stereotypes and the generation of compensatory and 
noncompensatory expectancies of group members. Personality and Social Psychology Bulletin, 19, 722-731. doi:10.1177/0146167293196007

Sharot, T., Korn, C. W., \& Dolan, R. J. (2011). How unrealistic optimism is maintained in the face of reality. Nature Neuroscience 4, 1475-1479. doi:10.1038/nn.2949

Sherman, J. W., Lee, A. Y., Bessenoff, G. R., \& Frost, L. A. (1998). Stereotype efficiency reconsidered: Encoding flexibility under cognitive load. Journal of Personality and Social Psychology, 75, 589-606. doi:10.1037/0022-3514.75.3.589

Shimkunas, A. M. (1970). Anxiety and expectancy change: The effects of failure and uncertainty. Journal of Personality and Social Psychology, 15, 34-42.

Spicer, S. G., Mitchell, C. J., Wills, A. J., \& Jones, P. M. (2020). Theory protection in associative learning: Humans maintain certain beliefs in a manner that violates prediction error. Journal of Experimental Psychology: Animal Learning and Cognition, 46, 151-161. doi:10.1037/xan0000225

Staples-Bradley, L. K., Treanor, M., \& Craske, M. G. (2018). Discrimination between safe and unsafe stimuli mediates the relationship between trait anxiety and return of fear. Cognition and Emotion, 32, 167-173. doi:10.1080/02699931.2016.1265485

Strelow, A. E., Dort, M., Schwinger, M., \& Christiansen, H. (2020). Influences on pre-service teachers' intention to use classroom-management strategies for students with ADHD: A model analysis. International Journal of Educational Research, 103, 101627. doi:10.1016/j.ijer.2020.101627

Strojny, P., Kossowska, M., \& Strojny, A. (2016). Search for expectancy-inconsistent information reduces uncertainty better: The role of cognitive capacity. Frontiers in Psychology, 7, 395. doi:10.3389/fpsyg.2016.00395

Süssenbach, P., Gollwitzer, M., Mieth, L., Buchner, A., \& Bell, R. (2016). Trustworthy tricksters: Violating a negative social expectation affects source memory and person perception when fear of exploitation is high. Frontiers in Psychology, 7, 2037. 
COPING WITH EXPECTATION VIOLATION 33

doi:10.3389/fpsyg.2016.02037

Vlaeyen J. W. S., \& Linton, S. J. (2000). Fear-avoidance and its consequences in chronic musculoskeletal pain: A state of the art. Pain, 85, 317-332. doi:10.1016/S03043959(99)00242-0

Willard, J. (2008). Understanding how shared expectations can shape reality: An examination of the underlying mechanisms in the accumulation of perceptual bias effects. Unpublished dissertation, Iowa State University.

Yanagisawa, H., \& Mikami, N. (2015). How does expectation change perception? A simulation model of expectation effect. In C. Weber, S. Husung, G. Cascini, M. Cantamessa, D. Marjanovic, \& M. Bordegoni (Eds.), Proceedings of the 20th International Conference on Engineering Design (pp. 149-158). Milan: ICED. 
Table 1

Predictors of Assimilation, Accommodation, and Immunization after the Experience of Expectation Violation

\begin{tabular}{|c|c|c|c|}
\hline Predictors & Accommodation & Assimilations & Immunization \\
\hline \multicolumn{4}{|l|}{ Characteristics of the expectation } \\
\hline Negative valence & - & & \\
\hline Certainty & - & & + \\
\hline Elaborateness & $-?$ & & $\pm^{1} ?$ \\
\hline $\begin{array}{l}\text { Consistence of previous } \\
\text { support }\end{array}$ & + & & - \\
\hline \multicolumn{4}{|c|}{ Characteristics of disconfirming information } \\
\hline Intensity of disconfirmation & $\begin{array}{c}\text { inverse u-shaped or } \\
+\end{array}$ & & u-shaped \\
\hline $\begin{array}{l}\text { Frequency of current } \\
\text { disconfirmation }\end{array}$ & + & & - \\
\hline Credibility & + & & - \\
\hline Unambiguousness & + & & - \\
\hline Valence: better than expected & + & & - \\
\hline Controllability & - & + & \\
\hline \multicolumn{4}{|c|}{ Characteristics of the broader situation } \\
\hline $\begin{array}{l}\text { Similarity of expectation to } \\
\text { those of relevant others }\end{array}$ & - & & + \\
\hline Cognitive load & & & $\pm^{2}$ \\
\hline $\begin{array}{l}\text { Prior coping: failed } \\
\text { immunization and/or } \\
\text { assimilation }\end{array}$ & + & & \\
\hline \multicolumn{4}{|l|}{ Personality characteristics } \\
\hline $\begin{array}{l}\text { Dispositional tenacious goal } \\
\text { pursuit }\end{array}$ & & + & \\
\hline $\begin{array}{l}\text { Need for structure/cognitive } \\
\text { closure }\end{array}$ & & $\pm^{3}$ & $\pm^{3}$ \\
\hline Need for consistency & - & & + \\
\hline Optimism & $\begin{array}{l}\text {-/0 (after worse- } \\
\text { than-exp. info.) }\end{array}$ & & + \\
\hline Neuroticism & $\begin{array}{l}\text { - (after better-than- } \\
\text { exp. information) }\end{array}$ & & \\
\hline Rejection/victim sensitivity & $\begin{array}{l}+ \text { (after better-than } \\
\text { expected info.) }\end{array}$ & & \\
\hline Trait anxiety & $\pm^{4}$ & & \\
\hline Depression & $\begin{array}{l}\text { - (after better-than- } \\
\text { exp. information) }\end{array}$ & & $\begin{array}{l}+ \text { (after better- } \\
\text { than-exp. info.) }\end{array}$ \\
\hline Metatrait Stability & $-?$ & $+?$ & $+?$ \\
\hline
\end{tabular}


Note. + indicates a positive association between the predictor and coping process, 0 indicates lack of observed association, ? indicates that this assumption has not yet been empirically tested. ${ }^{1}$ Negative association with non-detecting/ignoring, positive association with other forms of immunization. ${ }^{2}$ Depending on the amount of cognitive resources needed for immunizing. ${ }^{3}$ Depending on whether or not this coping process reduces uncertainty/ambiguity more than the other processes. ${ }^{4}$ Depending on the previous amount of support for the expectation. 